\title{
Laryngeal Sarcoidosis Causing Acute Airway Obstruction: A Rare Sequela of a Rare Entity
}

\author{
Shiksha Kedia ${ }^{\mathrm{a}, \mathrm{e}}$, Ashish Koirala ${ }^{\mathrm{b}}$, Vijaya Raj Bhatta, Sainath Gaddam ${ }^{\mathrm{a}}$, \\ Marina Landa ${ }^{c}$, Theodore Maniatis ${ }^{\mathrm{d}}$
}

\begin{abstract}
Laryngeal sarcoidosis, a rare entity, presents a diagnostic challenge and can masquerade as asthma. We present a case of a 53-year-old man with laryngeal sarcoidosis, which was initially misdiagnosed as asthma. The delay in the diagnosis resulted in an airway emergency and required an urgent tracheostomy.
\end{abstract}

Keywords: Sarcoidosis; Larynx; Airway obstruction

\section{Introduction}

Sarcoidosis, a multisystem disorder of unknown etiology characterized by the presence of noncaseating granulomas, involves larynx only in $1.2 \%$ of cases [1]. Because of its rarity, laryngeal sarcoidosis presents a diagnostic challenge. We present a case of a 53-year-old man with laryngeal sarcoidosis, which was initially misdiagnosed as asthma. The delay in the diagnosis resulted in an airway emergency and required an urgent tracheostomy.

Manuscript accepted for publication August 13, 2012

\footnotetext{
${ }^{a}$ Staten Island University Hospital, Department of Medicine, 475 Seaview Avenue, Staten Island, New York, USA

${ }^{\mathrm{b}}$ State University of New York Downstate Medical Center, Department of Medicine, Division of Pulmonary and Critical Care, 450 Clarkson Ave, Brooklyn NY, USA

${ }^{\mathrm{c} S t a t e n}$ Island University Hospital, Department of Pathology, 475 Seaview Avenue, Staten Island, New York, USA

${ }^{\mathrm{d}}$ Staten Island University Hospital, Department of Pulmonary and Critical Care, 475 Seaview Avenue, Staten Island, New York, USA

${ }^{\mathrm{e}}$ Corresponding author: Shiksha Kedia, Staten Island University

Hospital, Department of Internal Medicine, 475 Seaview Avenue, Staten Island, New York, 10305, USA.

Email: shiksha.kedia@gmail.com
}

doi: http://dx.doi.org/10.4021/jmc831w

\section{Case Report}

A 53-year-old African-American man presented to the emergency room with complaints of worsening shortness of breath and dry cough since last few weeks. He also complained of hoarseness and intermittent stridor since last several days. The patient denied fever, chills, night sweats, rash, joint pain, blurring of vision or abdominal pain. Past medical and surgical history was only significant for Type II Diabetes mellitus. His shortness of breath and intermittent wheezing was attributed to asthma for five years by his primary physician. Home medications included metformin, montelukast, inhaled salmeterol/fluticasone and inhaled albuterol as needed. Family history was insignificant for sarcoidosis, respiratory illness or any malignancies. He had a smoking history of 10 pack-years but denied any drinking of alcohol, illicit drug use, recent travel or contact with tuberculosis patient.

On examination, he had a respiratory rate of 26 per minute, temperature of 97.3 degree Fahrenheit, blood pressure of 143/84 mm of mercury, heart rate of 109 beats per minute and oxygen saturation of $96 \%$ on room air. He had significant stridor and hoarseness and was not able to speak in complete sentences. There was no pallor, icterus, clubbing, cyanosis or palpable lymphadenopathy. Throat examina-

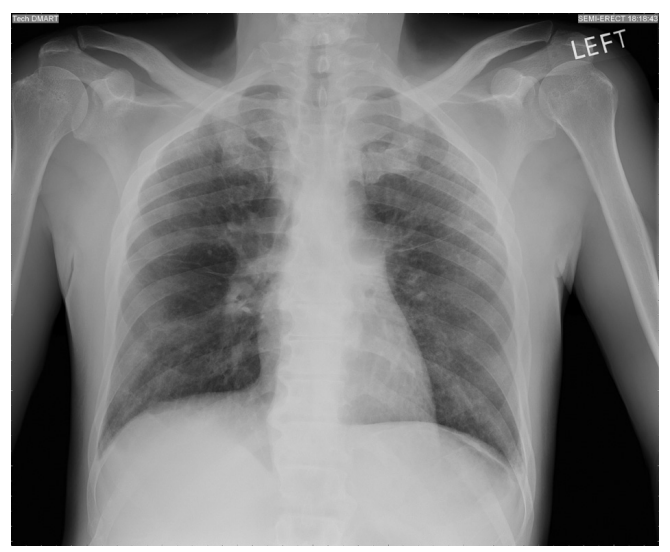

Figure 1. Chest X-ray showing non-specific interstitial edema. 


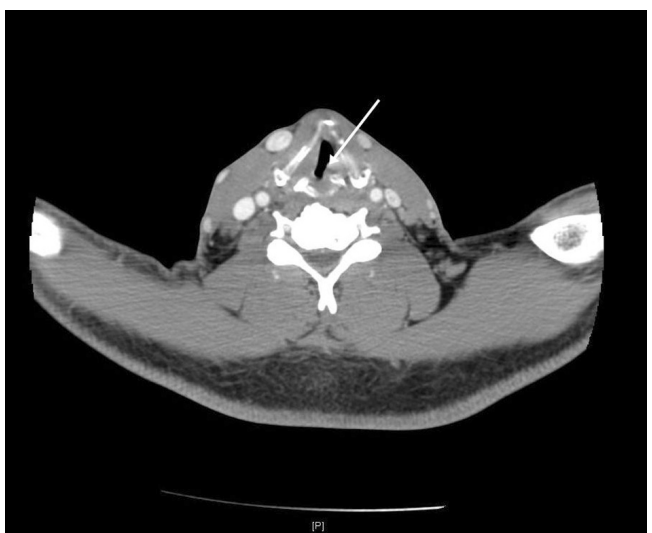

Figure 2. Computed Tomography Scan of the neck (Axial view) showing submucosal infiltration of the supraglottic larynx and left vocal cord fixation.

tion was normal with normal looking tonsils and undisplaced uvula. Chest auscultation revealed diminished but bilateral equal breath sounds and rhonchi. Rest of the examination was unremarkable.

Laboratory studies included: white blood cell count of $5900 / \mu \mathrm{l}$ with normal differential, hemoglobin of $15.9 \mathrm{~g} / \mathrm{dl}$, platelet count of $199,000 / \mu \mathrm{l}$, calcium of $8.8 \mathrm{mg} / \mathrm{dl}$, erythrocyte sedimentation rate of $4 \mathrm{~mm} / \mathrm{hr}, \mathrm{C}$-reactive protein of $<$ $0.5 \mathrm{mg} / \mathrm{dl}$, and angiotensin converting enzyme level of 51 U/L. Liver and renal function tests and test for Wegener's granulomatis were all within normal limits. He had a negative result on Purified Protein Derivative (PPD) skin testing. Chest X-ray revealed non-specific interstitial edema (Fig. 1). A computed tomography (CT) of the soft tissues of the neck with intravenous contrast showed sub-mucosal infiltration of

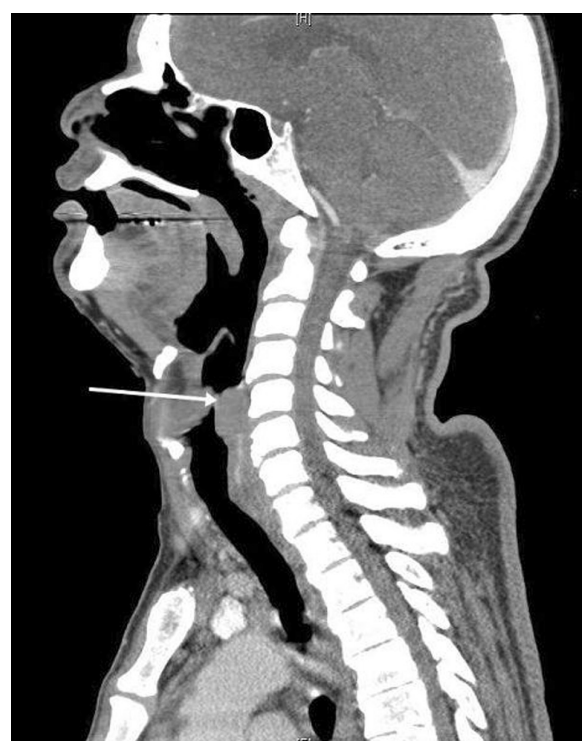

Figure 3. Computed Tomography Scan of the neck (Sagittal reconstruction) showing acute airway narrowing.

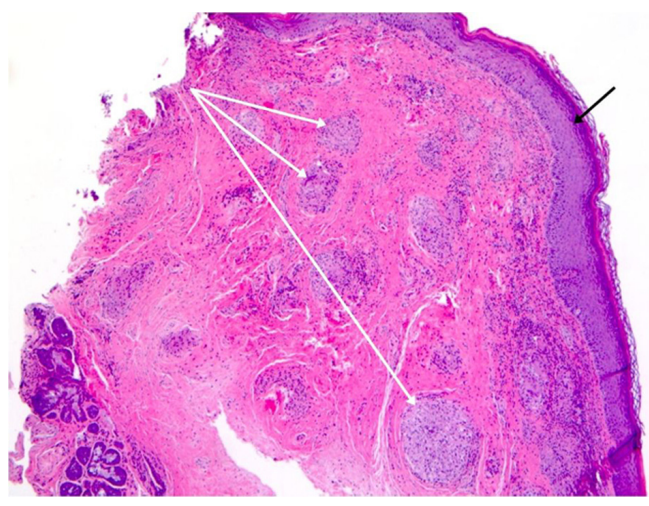

Figure 4. Histopathology of the supraglottis mass (low power) showing squamous epithelium (black arrow) and underlying soft tissue showing multiple non-caseating granuloma (white arrow).

the left supraglottic larynx with possible vocal cord fixation and airway narrowing (Fig. 2, 3). It also showed mediastinal lymphadenopathy and enlarged lymph nodes at the base of neck. A flexible fiberoptic laryngoscopy revealed a left supraglottic sub-mucosal, non-exophytic mass, located over left arytenoid causing airway obstruction. The left true vocal cord was medialized and paralysed. The right vocal cord was normal. With the diagnosis of impending upper airway obstruction, an urgent tracheostomy was performed under general anesthesia and the patient was monitored in the Intensive Care Unit. Systemic steroid therapy was started with improvement in the patient's condition. Histopathology examination of the paratracheal lymph nodes and supraglottic mass showed non-caseating granulomas with few giant cells (Fig. 4, 5). Special stains for fungi and tuberculosis were negative. Thus, a diagnosis of laryngeal sarcoidosis with acute airway obstruction was confirmed.

\section{Discussion}

First described by Hutchinson in 1877, sarcoidosis is a mul-

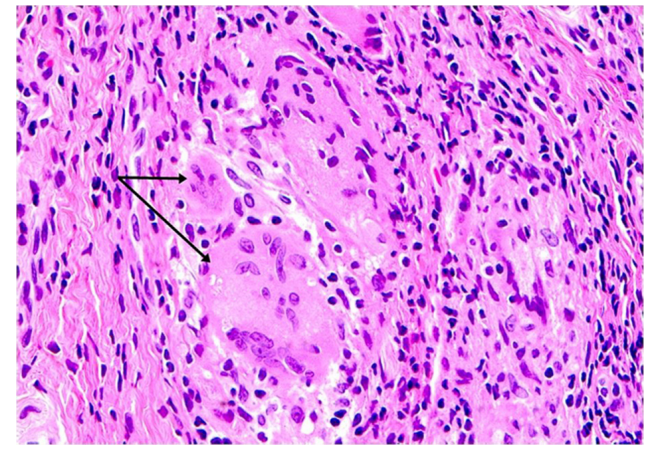

Figure 5. Histopathology of the supraglottis mass (high power) showing Langhans cells. 
tisystem disorder characterized by the presence of non-caseating granulomas and affects young adults aged $20-40$ years old, women more than men and black more than white [2]. It mainly involves the pulmonary system and is one of the few diseases which can involve any part of the respiratory tract from the nose to respiratory bronchioles [3]. Upper airway is involved only in $6 \%$ of cases with laryngeal involvement seen in rare $1.2 \%$ [1].

Laryngeal sarcoidosis more commonly involves supraglottic area including epiglottis, arytenoids and false vocal cords because of its rich lymphatic supply. On the other hand, true vocal cords and subglottic region are rarely involved [47]. Laryngeal sarcoidosis usually presents with dysphonia, irritating dry cough and dysphagia $[4,8]$. Pharyngolaryngeal discomfort, globus pharyngus, hyponasal tone can be some of the other presenting symptoms. Obstructive sleep apnea is seen in $5 \%$ of upper airway disease. In most circumstances the disease remits spontaneously and has a benign course. However, as illustrated by the case, laryngeal involvement can potentially lead to life threatening airway obstruction [4]. Extrinsic compression of airway by enlarged lymph nodes as well as inflammation resulting in mucosal edema and granuloma formation are the mechanisms responsible for airway obstruction [4-7].

A definitive diagnostic test for sarcoidosis does not exist. Therefore diagnosis requires the presence of compatible clinical, radiographic, and histopathologic findings and the exclusion of other possible disease processes [2]. Isolated laryngeal sarcoidosis presents further diagnostic challenges [9, 10]. Early symptoms are nonspecific and the disease is rare. Thus, physicians might falsely attribute early symptoms to more common disease processes such as asthma as it happened in our patient. Furthermore, it has to be differentiated from several other diseases such as relapsing polycondritis, Wegener's granulomatosis, papillomatosis, non-specific mucosal granulomas, amyloidosis, histoplasmosis, blastomycosis, tuberculosis, syphilis, lymphoma, squamous cell carcinoma and cartilaginous tumors. These conditions share many of the manifestations of laryngeal sarcoidosis and require exclusion [1]. However, it is important to establish an early diagnosis to prevent potential complication such as airway obstruction [11].

Fibreoptic laryngoscopy may reveal pale, edematous and diffusely enlarged supraglottis, with nodules, ulcerations, polypoid and granulomatous mass. Involvement of the rim of epiglottis may produce a turban like appearance which is pathognomonic for sarcoidosis $[12,13]$. Computed tomography (CT) of the soft tissues of the neck reveals enlargement of the epiglottis, thickening of the vocal cords and subglottic stenosis and has been used widely to define the anatomy, and detect masses and upper airway narrowing [6]. Histopathology shows epitheloid non-caseating granulomas with multinucleated giant cells that contain asteroid bodies, schaumann bodies and birefringent crystalline material [14-
16]. Presence of these findings in the appropriate clinical context and exclusion of other conditions can help establish the diagnosis.

Treatment varies with the clinical scenario, ranging from close monitoring in stable asymptomatic patients to emergent tracheostomy in patients with acute airway obstruction [5]. Systemic steroid therapy is the mainstay of therapy for a systemic disease. Treatment modalities for localized upper airway disease include carbon dioxide $\left(\mathrm{CO}_{2}\right)$ laser treatment or inhaled or intralesional steroids [5, 17, 18]. Combined modalities may include intralesional steroids with mucosa sparing laser surgery or laser surgery and the application of mitomycin-C $[19,20]$. Cytotoxic agents such as methotrexate and azathioprine, antimalarials such as chloroquine and hydroxychloroquine, external beam radiation therapy and surgical excision have also been used [21,22]. Racemic epinephrine can also be tried in acute situations which may lead to vasoconstriction and reduction of edema [4, 23]. Although close monitoring is an option in stable patients, early therapy is particularly important in symptomatic progressive disease to prevent serious complication.

Overall prognosis of laryngeal sarcoidosis is good. According to a study at Mayo clinic in 1982, only few cases required prolonged tracheostomy while others recovered with conservative management [18]. Similar results were shown in a case review of laryngeal sarcoidosis with bilateral vocal cord paralysis [24].

In conclusion, laryngeal sarcoidosis presents with nonspecific symptoms. It can masquerade other common diseases such as asthma and present diagnostic challenge. Delay in diagnosis and therapy can result in airway obstruction with consequent morbidity and potentially mortality.

\section{Grant}

None.

\section{Competing Interests}

The authors declare that they have no competing interests.

\section{References}

1. Polychronopoulos VS, Prakash UB. Airway involvement in sarcoidosis. Chest. 2009;136(5):1371-1380.

2. Statement on sarcoidosis. Joint Statement of the American Thoracic Society (ATS), the European Respiratory Society (ERS) and the World Association of Sarcoidosis and Other Granulomatous Disorders (WASOG) adopted by the ATS Board of Directors and by the ERS Executive Committee, February 1999. Am J Respir Crit Care 
Med. 1999;160(2):736-755.

3. Morgenthau AS, Teirstein AS. Sarcoidosis of the upper and lower airways. Expert Rev Respir Med. 2011;5(6):823-833.

4. Davis C, Girzadas DV, Jr. Laryngeal sarcoidosis causing acute upper airway obstruction. Am J Emerg Med. 2008;26(1):114 e111-113.

5. Dean CM, Sataloff RT, Hawkshaw MJ, Pribikin E. Laryngeal sarcoidosis. J Voice. 2002;16(2):283-288.

6. Ferretti GR, Calaque O, Reyt E, Massot C, Coulomb M. CT findings in a case of laryngeal sarcoidosis. Eur Radiol. 2002;12(4):739-741.

7. Devine KD. Sarcoidosis and Sarcoidosis of the Larynx. Laryngoscope. 1965;75:533-569.

8. Poe DL. Sarcoidosis of the larynx. Arch Otolaryngol.1940; 32(2):315-20.

9. Israel HL, Sones M. Sarcoidosis; clinical observation on one hundred sixty cases. AMA Arch Intern Med. 1958;102(5):766-776.

10. Herlow LB, Rasmussen N. [Laryngeal sarcoidosis in 13 year-old teenager]. Ugeskr Laeger. 2010;172(45):31163117.

11. Mayerhoff RM, Pitman MJ. Atypical and disparate presentations of laryngeal sarcoidosis. Ann Otol Rhinol Laryngol. 2010;119(10):667-671.

12. Carasso B. Sarcoidosis of the larynx causing airway obstruction. Chest. 1974;65(6):693-695.

13. Weiss JA. Sarcoidosis in otolaryngology. Report of eleven cases. Evaluation of blind biopsy as a diagnostic aid. Laryngoscope. 1960;70:1351-1398.

14. Kitaichi M. Pathology of pulmonary sarcoidosis. Clin Dermatol. 1986;4(4):108-115.

15. Longcope WT, Freiman DG. A study of sarcoidosis; based on a combined investigation of 160 cases includ- ing 30 autopsies from The Johns Hopkins Hospital and Massachusetts General Hospital. Medicine (Baltimore). 1952;31(1):1-132.

16. Myers JL, Tazelaar HD. Challenges in pulmonary fibrosis: 6--Problematic granulomatous lung disease. Thorax. 2008;63(1):78-84.

17. Ruff T, Bellens EE. Sarcoidosis of the larynx treated with CO2 laser. J Otolaryngol. 1985;14(4):245-247.

18. Plaschke CC, Owen HH, Rasmussen N. Clinically isolated laryngeal sarcoidosis. Eur Arch Otorhinolaryngol. 2011;268(4):575-580.

19. James JC, Simpson CB. Treatment of laryngeal sarcoidosis with CO2 laser and mitomycin-C. Otolaryngol Head Neck Surg. 2004;130(2):262-264.

20. Butler CR, Nouraei SA, Mace AD, Khalil S, Sandhu SK, Sandhu GS. Endoscopic airway management of laryngeal sarcoidosis. Arch Otolaryngol Head Neck Surg. 2010;136(3):251-255.

21. Gerencer RZ, Keohane JD, Jr., Russell L. Laryngeal sarcoidosis with airway obstruction. J Otolaryngol. 1998;27(2):90-93.

22. Fogel TD, Weissberg JB, Dobular K, Kirchner JA. Radiotherapy in sarcoidosis of the larynx: case report and review of the literature. Laryngoscope. 1984;94(9):12231225.

23. Davis AM, Punniyamoorthy S, Griffin AM, Wunder JS, Bell RS. Symptoms and their Relationship to Disability Following Treatment for Lower Extremity Tumours. Sarcoma. 1999;3(2):73-77.

24. Coffey CS, Vallejo SL, Farrar EK, Judson MA, Halstead LA. Sarcoidosis presenting as bilateral vocal cord paralysis from bilateral compression of the recurrent laryngeal nerves from thoracic adenopathy. J Voice. 2009;23(5):631-634. 